\title{
Model-based control of slugging flow: an experimental case study
}

\author{
Florent Di Meglio, Glenn-Ole Kaasa, Nicolas Petit, Vidar Alstad
}

\begin{abstract}
In this paper, we compare two solutions for suppressing slug flow in pipes conveying a diphasic fluid by active feedback control of the outlet (production) choke. Conventionnaly, a non-collocated PI controller is used to stabilize the measured inlet pressure. Unfortunately, this method lacks robustness to changes in operating conditions and reveals difficult to calibrate. In this work, we propose a model-based approach to design a nonlinear state-feedback control law based on a first principles model of the slugging phenomenon. A theoretical proof of convergence of the closed-loop model is provided, as well as an experimental validation of these results on a mid-scale testbench.
\end{abstract}

\section{INTRODUCTION}

In this article, we study means to suppress slug flow by closed-loop control. Slug flow is a hydrodynamic regime, occuring in numerous industrial situations, mostly in onedimensionnal pipes conveying a diphasic medium (gas and liquid). This regime is characterized by oscillatory pressures and flow rates, due to the inhomogenous spatial distribution of the two phases. In this regime, elongated bubbles of gas separated by "slugs" of liquid travel from one end of the pipe to the other. The occurence of the slug flow regime is frequent during the oil extraction process. In particular, risers, which are long pipes connecting reservoirs or seabed installations to surface facilities, are subjected to this type of flow. The formation of these inhomogeneities has been widely studied [1], [2], [3], [4], [5]. While numerous cases are relatively well understood (risers with low-point [6], [7], gas-lifted systems [8], [9]), some important occurences of practical interest have yet to be explained.

Slug flow generates a decrease in the average flow output, which can easily be as large as 50\%. This relative inefficiency results in substantial profit losses. Further, the pressure variations induced by the slug flow may damage the facilites. For these two reasons, ways of suppressing the slug flow are of paramount importance. What is desired is a homogeneous steady flow where very small bubbles of gas are well distributed in the continuous liquid phase. In this desired situation, the production reaches its nominal value, and the pressure remains constant over time.

In practice, input and output chokes can serve as actuators for the riser. Besides, pressure sensors (which can be complemented by density sensors) can be used to determine

F. Di Meglio (corresponding author) is a $\mathrm{PhD}$ candidate in Mathematics and Control at Mines Paristech, 60, Bd St-Michel, 75272 Paris, Cedex 06, France

G.O. Kaasa and Vidar Alstad are Research Engineer at StatoilHydro ASA, Research Center Porsgrunn, Heroya Forksningspark 3908 Porsgrunn, Norway

N. Petit is Professor at Mines Paristech, 60, Bd St-Michel, 75272 Paris, Cedex 06, France the appropriate control actions. A prime example of possible way to suppress the slug flow is, as has been proposed in [10], to use a feedback PI controller, using the downside pressure measurement (often referred to as Riser Base Pressure (RBP)) to actuate the outlet choke. This non-collocated control strategy has proven very efficient in a great number of applications [11], [12], [13]. Yet, this efficiency can be largely improved. Some of the relative flaws of this method are its lack of robustness, frequent need of retuning and overall unsufficient reliability.

In an attempt to address the preceding issues, we propose to follow a model-based approach to design a better suited control scheme. As will appear, analysis of a firstprinciples model (which we first presented in [14]) stresses the importance of a state variable closely related (but not equivalent) to the RBP, namely the total mass of liquid in the riser. Following ideas from [6] and [9], the model consists of ordinary differential equations (ODE) where the state variables are two masses of gas and the mass of liquid mentionned above, while the control variable is the opening of the production outlet choke. It reproduces the oscillations observed during the slugging phenomenon, which here correspond to an unstable equilibrium point. Several nonlinearities appear in the right-hand side of the equations, yet, this model is stabilizable by a straightforward partially linearizing feedback, which is, along with its theoretical proof of convergence, and its practical implementation, the first contribution of this paper.

A second contribution is a comparison of the performances of the two discussed approaches. Using the Statoil experimental facilities located in Porsgrunn, Norway, we illustrate the merits of the model-based proposed control law, and compare it against a reference PI controller. The Statoil multiphase flow loop is designed to reproduce the behavior of offshore oil wells and flowlines, where the slugging phenomenon commonly arises. An identification of the model parameters is necessary. Then, it appears that this nonlinear control law can handle a vast range of operating conditions. In particular, large production choke opening values can be reached. These lie in unstable domains that could not be stabilized by the reference controller. Transposed on a real system, this improvement should have a direct impact on the level of production, and thus on the profitability of the facility.

The paper is organized as follows. In Section II, we present the problem under consideration, as well as the state-of-the-art solution. Then, we recall in Section III the main principles of the model introduced in [14] and new developments. Further, we present in Section IV the control 
law we derived from this model, as well as the theoretical proof of convergence of the closed-loop system. Eventually, we present the experimental validation of this result in Section $\mathrm{V}$, along with a comparison with the performances of the reference PI controller.

\section{Problem DESCRIPTION}

\section{A. Slug flow}

Two classes of systems are subject to the slug flow regime: wells and flowlines. Wells directly connect the reservoirs to seabed or surface facilites. They are characterized by a long (up to $\approx 3500 \mathrm{~m}$ ) upward inclined section referred to as the riser, sometimes preceded by a horizontal section. Flowlines, on the other hand, gather the production coming from several wells at the seabed, and transport it to the surface facilities. They are composed of a long, nearlyhorizontal section (several thousand meters) followed by a shorter $(\approx 200 \mathrm{~m})$ vertical riser. In the rest of the article, our main concern will be the dynamics of the oil and the gas inside the riser, which can be part either of a well or a flowline. Althought the characteristics of the slugging are different for both classes of systems, our model is able to reproduce a range of behaviors large enough to cover them all.

The slug flow regime arises mostly when entering tail production of an oil field. It is characterized by an unstable multiphase flow, where "slugs" of liquid accumulate before being pushed upwards by the gas. It is also characterized by oscillations of the pressure in the pipe and oscillations of flow rates of gas and oil at the production end of the pipe. Under its most severe form, the slugging flow regime can lead to unwanted shutdowns and wear and tear on equipment and, most importantly, reduce the oil production. For these reasons, various techniques have been investigated in view of suppressing it.

The first solution to arise was the manual choking of the pipe. Early studies [15], [16] showed that reducing the opening of the production choke could stabilize the flow, thus eliminating slugging. Storkaas [6] identified this behavior as a Hopf bifurcation: as one reduces the choke opening, the unstable poles of the system cross the imaginary axis and become stable. Unstable poles correspond to the slugging behavior, whereas stable ones correspond to steady flows, namely bubbly or annular. The point were the eigenvalues are purely complex is called the bifurcation point. The big drawback of reducing the choke opening is that it increases the backpressure in the pipe, which reduces the production of oil. The control challenge is therefore to stabilize the flow while keeping a low backpressure, that is to say, suppress the slugging around an operating point that is unstable with manual control of the choke.

\section{B. Active feedback control}

The most efficient way to cope with slugging was found to be active feedback control of the production choke [12], [17], [18]. A common solution consists in applying a PI controller using the Riser Base Pressure (RBP) as the measured output.
This technique has proven very efficient in improving the oil recovery in a great number of applications [6], [10], [19], [20]. Still, its efficiency can be largely improved in three different aspects:

a) Necessity of downside measurement: Storkaas [6] showed that the RBP was a much better suited variable to control with a PI than the topside pressure. This justified the already widely spread use of the RBP to stabilize slugging oil fields. Yet, even though this measurement is usually available on flowlines, it is not always the case on wells, for which it is much more difficult to install. Topside measurements, by contrast, are very easily available. Moreover, they are usually much more reliable than the downside measurements.

b) Sensitivity to variations: Petroleum engineers have experienced that the PI controller had to be frequently retuned to remain efficient. Specifically, a small change in the operating conditions (e.g. due to a depletion of the reservoir) could cause the controller to fail in stabilizing the flow, and require the retuning of the gains. In other words, the range of values of the gains yielding stabilization is very narrow.

c) Poor improvement of the oil recovery: None of the PI controllers presented in the litterature have been able to stabilize an unstable operating point far from the bifurcation point, that is to say with a low backpressure. In order to significantly reduce the production losses due to slugging, a controller should be able to stabilize the flow for higher choke openings, where the instability is more difficult to cope with.

d) Sensitivity to the state of the system: The slugging phenomenon consists in periodic oscillations of the pressure. Experimentally, it has been observed that the efficiency of the PI controller depends highly on the stage of this cycle when it is turned on. More specifically, it has been seen that one needs to turn the controller on when the values of the RBP start decreasing in order for it to stabilize the flow. This behavior, which is obviously problematic, shows a dependency on variables (states) that are not accounted for in the control scheme of the PI controller.

\section{The MOdel: A SHORT REMINDER AND EXTENSIONS}

Our approach consists in using a mathematical model to elaborate a controller well suited to the slugging problem. A first attempt in this direction was made by Eikrem et al. in [21], but the litterature shows no evidence of their results being applied to an experimental case. The basis of the model we used were described in [14], and, for convenience, we give in this section a brief reminder of its main principles. Then, we present new developments and, in the next section, the model-based control law which can be derived from it.

\section{A. Review of the model}

The system of interest is a transportation line with a riser, either a flowline or a well. The geometry of this transportation line can be very general, in particular, the existence of a low-point is not required but can be considered. Yet, the model assumes the existence of an obstructing liquid interface at a certain location in the riser, preventing 
the gas from flowing normally, and modelled by a valve equation. This yields the formation of an elongated bubble upstream this "virtual valve", where a build-up of pressure will occur and eventually generate instability. These features are depicted in Figure 1. The existence of this valve-like

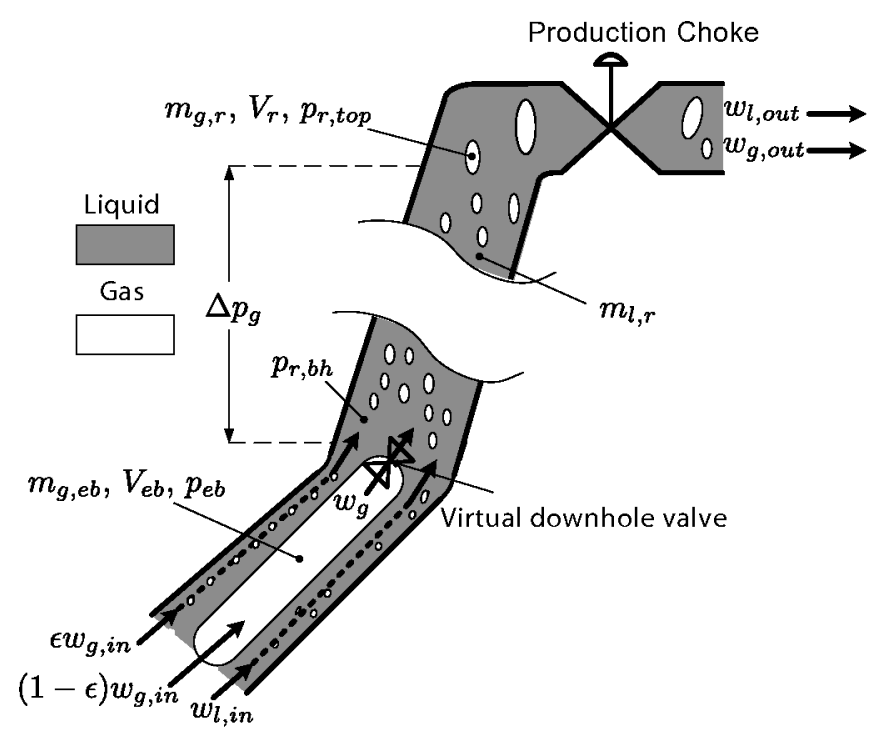

Fig. 1. Schematic representation of the transport line under consideration

separation was very well-identified by Storkaas in the case of risers with a low-point [6], and by Jansen et al. in the case of gas-lifted wells [18]. The idea of our model is simply to extend this concept to systems where the causes of its existence are not as clear, but the consequences - the slugging phenomenon - are observed. We now present the model, based on the conservation laws expressed for three masses: the mass of gas in the elongated bubble $\mathbf{m}_{\mathbf{g}, \mathbf{e b}}$, the mass of gas downstream a virtual valve $\mathbf{m}_{\mathbf{g}, \mathbf{r}}$, and the mass of liquid in the riser $\mathbf{m}_{\mathbf{l}, \mathrm{r}}$. For more details, the reader can refere to [14].

$$
\begin{aligned}
\dot{\mathbf{m}}_{\mathbf{g}, \mathbf{e b}} & =(1-\epsilon) w_{g, i n} \\
& -C_{g} \max \left[\frac{R T}{M V_{e b}} \mathbf{m}_{\mathbf{g}, \mathbf{e b}}-\frac{\rho_{l} R T}{M} \frac{\mathbf{m}_{\mathbf{g}, \mathbf{r}}}{m_{l}^{\Delta}-\mathbf{m}_{\mathbf{l}, \mathbf{r}}}\right. \\
& \left.-\left(\mathbf{m}_{\mathbf{l}, \mathbf{r}}+m_{l, s t i l l}\right) \frac{g \sin \theta}{A}, 0\right] \\
\dot{\mathbf{m}}_{\mathbf{g}, \mathbf{r}} & =\epsilon w_{g, i n} \\
& +C_{g} \max \left[\frac{R T}{M V_{e b}} \mathbf{m}_{\mathbf{g}, \mathbf{e b}}-\frac{\rho_{l} R T}{M} \frac{\mathbf{m}_{\mathbf{g}, \mathbf{r}}}{m_{l}^{\Delta}-\mathbf{m}_{\mathbf{l}, \mathbf{r}}}\right. \\
& \left.-\left(\mathbf{m}_{\mathbf{l}, \mathbf{r}}+m_{l, s t i l l}\right) \frac{g \sin \theta}{A}, 0\right] \\
& -\frac{\mathbf{m}_{\mathbf{g}, \mathbf{r}}}{\mathbf{m}_{\mathbf{l}, \mathbf{r}}} u C_{c} \sqrt{\frac{\rho_{l} R T}{M} \frac{\mathbf{m}_{\mathbf{g}, \mathbf{r}}}{m_{l}^{\Lambda}-\mathbf{m}_{\mathbf{l}, \mathbf{r}}}-p_{s}} \\
\dot{\mathbf{m}}_{\mathbf{l}, \mathbf{r}} & =w_{l, i n}-u C_{c} \sqrt{\frac{\rho_{l} R T}{M} \frac{\mathbf{m}_{\mathbf{g}, \mathbf{r}}}{m_{l}^{\Delta}-\mathbf{m}_{\mathbf{l}, \mathbf{r}}}-p_{s}}
\end{aligned}
$$

For sake of clarity, the scalar state variables $\mathbf{m}_{\mathbf{g}, \mathbf{e b}}, \mathbf{m}_{\mathbf{g}, \mathbf{r}}$ and $\mathbf{m}_{\mathbf{l}, \mathrm{r}}$ are in bold faces. The terms of the form $\max (\cdot, 0)$ represent the flow through a virtual valve, where no backflow is permitted. The term $\frac{R T}{M V_{e b}} \mathbf{m}_{\mathbf{g}, \mathbf{e b}}$ represents the pressure in the elongated bubble, the volume of which $V_{e b}$ is assumed constant. The term $\frac{\rho_{i} R T}{M} \frac{\mathbf{m}_{g, r}}{m_{l}^{\Delta}-\mathbf{m}_{1, \mathrm{r}}}$ represents the pressure at the top of the riser. It is given by the ideal gas law expressed for the mass of gas in the riser, the volume of which depends on the mass of liquid $\mathbf{m}_{1, \mathbf{r}}$ that compresses the gas. Besides, the pressure right after the virtual valve is computed by adding the pressure at the top of the riser to the gravity effect of the mass of liquid $\left(\mathbf{m}_{1, \mathbf{r}}+m_{l, s t i l l}\right) \frac{g \sin \theta}{A}$, where $\theta$ is the average inclination of the riser. The role of the term $m_{l, s t i l l}$, representing a "still" mass of liquid, will be discussed more in details in the next section. Eventually, the term under the square root represents the flow of liquid through the production choke. One should notice that the separator pressure $p_{s}$ (downstream the production choke) is assumed to be constant, and that the outflow of gas is simply computed from the outflow of liquid, mutliplied by the mass fraction $\frac{\mathbf{m}_{\mathrm{g}, \mathrm{r}}}{\mathbf{m}_{\mathrm{l}, \mathrm{r}}}$.

As shown in [14], this model is able to reproduce the oscillations of pressure and production flow rates corresponding to the slugging phenomenon, by fitting four parameters of the model using simple tuning rules. We now quickly present the latest improvements made to this model.

\section{B. Model improvements}

e) Still mass of liquid: We introduced the parameter $m_{l, \text { still }}$ in [14] as a possible addition to the model. It actually revealed crucial to allow the model to reproduce both the behavior of wells and that of flowlines. It allows to account for the fact that only a fraction of the total mass of liquid oscillates. Another way to see this is that the riser is not necessarily fully emptied during a slugging cycle. It can be the case, especially for severe slugging (typical of flowlines), where the riser is usually almost empty after the blowout phase, but for many other systems, (mostly wells), the model needs to reflect that there is a mass of liquid constantly weighting on the bottom of the riser. These considerations appear in the model at two places: first, the effect on the gravity pressure drop over the riser is represented by the term $m_{l, s t i l l} \frac{g \sin \theta}{A}$. Also, this still mass of liquid reduces the volume being available for the gas in the riser, which is now equal to $\frac{m_{l}^{\lambda}-m_{l, r}}{\rho_{l}}=\frac{m_{i}^{*}-m_{l, s i i l}-m_{l, r}}{\rho_{i}}\left(m_{l}^{*}\right.$ corresponds to a riser entirely filled with liquid).

The fraction of liquid that oscillates, or, equivalently, the fraction of liquid that remains in the riser, depends on the operating point under consideration. For sake of simplicity, we choose to keep it constant. Therefore, this parameter has to be tuned offline, before the model can be used. The way we perform this tuning is described in [14]: while the $C_{c}$ parameter is used to match the equilibrium value of the upstream choke pressure of our model with that of the system, the $m_{l, s t i l l}$ parameter is used to fit the equilibrium value of the pressure at the base of the riser.

f) Nonlinear valve equations: Formerly, we considered using valve equations of the type $w_{\text {out }}=C(\rho \Delta p)^{1 / n}$, which is a generalization of the conventional flow rate equation for an 
incompressible fluid and turbulent flow for $n=2$, and laminar flow for $n=1$. Even though the impact of this type of relation on the behavior of the model is never neglictible, the best results are always obtained using a value of $n$ very close to 2 . To limit the number of tuning parameters, we chose to keep $n=2$ in all future works.

\section{Control LaW}

In this section, we elaborate a control law based on the properties of the model. We show that it is sufficient to stabilize one of its variables, the mass of liquid in the riser, to stabilize the whole system. The proof is based on a classic Lyapunov analysis. We will assume that a full state is available for feedback. In practice, this actually requires the use of an observer, as the masses of gas and liquid cannot be measured. A high-gain type of observer, using only the topside measurement, was developped for this model and used in experimentations.

To simplify the following computations, we rewrite the system (1)-(2)-(3) using a new set of coordinates $\left(x_{1}, x_{2}, x_{3}\right)=\left(m_{g, e b}, \frac{m_{g, r}}{m_{l, r}}, m_{l, r}\right)$. This gives us the following system

$$
\begin{gathered}
\dot{x}_{1}=(1-\epsilon) w_{g, \text { in }}-C_{g} \max \left(a x_{1}-b \frac{x_{2} x_{3}}{m_{3}^{\Delta}-x_{3}}\right. \\
-\left(x_{3}+m_{3, \text { still })} \frac{g \sin \theta}{A}, 0\right) \\
\dot{x}_{2}=\frac{1}{x_{3}}\left[\epsilon w_{g, \text { in }}+C_{g} \max \left(a x_{1}-b \frac{x_{2} x_{3}}{m_{3}^{\Delta}-x_{3}}\right.\right. \\
\left.\left.-\left(x_{3}+m_{3, \text { still }}\right) \frac{g \sin \theta}{A}, 0\right)-x_{2} w_{l, \text { in }}\right] \\
\dot{x_{3}}=w_{l, \text { in }}-u C_{c} \rho_{l}\left(b \frac{x_{2} x_{3}}{m_{3}^{\Delta}-x_{3}}-p_{s}\right)^{1 / n}
\end{gathered}
$$

where the constants $a=\frac{R T}{M V_{e b}}$ and $b=\frac{\rho_{l} R T}{M}$ are introduced for sake of clarity. We also denote

$$
y=b \frac{x_{2} x_{3}}{m_{3}^{\Delta}-x_{3}}
$$

the measured output of the system. For any given choke opening $0<\bar{u}<1$, the system has a unique corresponding equilibrium point $\left(\bar{x}_{1}, \bar{x}_{2}, \bar{x}_{3}\right)$ (full expressions are given in appendix). Conversely, for any given $0<\bar{x}_{3}<\frac{\bar{y}(1)}{\bar{y}(1)+b \frac{w_{g, i n}}{w_{l, i n}}} m_{3}^{\Delta}$, there is a unique corresponding equilibrium choke opening $\bar{u}$.

Theorem 4.1: The partially linearizing feedback law

$$
u(t)=\frac{w_{l, i n}+k\left(x_{3}(t)-\bar{x}_{3}\right)}{C_{c} \rho_{l}\left(y(t)-p_{s}\right)^{1 / n}}
$$

with $k>0$ stabilizes the system around its equilibrium point $\left(\bar{x}_{1}, \bar{x}_{2}, \bar{x}_{3}, \bar{u}\right)$ which is Locally Asymptotically Stable. A conservative estimation of the basin of attraction is constructed in the proof.

Proof: First, we consider the open set $D^{+}=$ $\left\{a x_{1}-b \frac{x_{2} x_{3}}{m_{3}^{\Delta}-x_{3}}-\left(x_{3}+m_{3, \text { still }}\right) \frac{g \sin \theta}{A}>0\right\}$ where the expression inside the max functions is strictly positive. We also introduce the error variables $\tilde{x}_{i}=x_{i}-\bar{x}_{i}$. In $D^{+}$, the closed-loop system yields the following cascaded error dynamics

$$
\begin{aligned}
\dot{\tilde{x}}_{1} & =-C_{g} a \tilde{x}_{1}+C_{g} \frac{\bar{y}}{\bar{x}_{2}} \tilde{x}_{2} \\
& +\tilde{x}_{3}\left[C_{g} \frac{g \sin \theta}{A}+b \frac{\tilde{x}_{2}+\bar{x}_{2}}{\left(m_{3}^{\Delta}-\bar{x}_{3}-\tilde{x}_{3}\right)\left(m_{3}^{\Delta}-\bar{x}_{3}\right)}\right] \\
\dot{\tilde{x}}_{2} & =\frac{C_{g} a}{\bar{x}_{3}} \tilde{x}_{1}-\frac{1}{\bar{x}_{3}}\left(w_{l, i n}+\frac{C_{g} \bar{y}}{\bar{x}_{2}}\right) \tilde{x}_{2} \\
& -\frac{\tilde{x}_{3}}{\bar{x}_{3}\left(\bar{x}_{3}+\tilde{x}_{3}\right)}\left[C_{g} a \tilde{x}_{1}-\left(w_{l, i n}+\frac{C_{g} \bar{y}}{\bar{x}_{2}}\right) \tilde{x}_{2}\right] \\
& +\frac{\tilde{x}_{3}}{\left(\bar{x}_{3}+\tilde{x}_{3}\right)}\left[C_{g} \frac{g \sin \theta}{A}+b \frac{\tilde{x}_{2}+\bar{x}_{2}}{\left(m_{3}^{\Delta}-\bar{x}_{3}-\tilde{x}_{3}\right)\left(m_{3}^{\Delta}-\bar{x}_{3}\right)}\right] \\
\dot{\tilde{x}}_{3} & =-k \tilde{x}_{3}
\end{aligned}
$$

We now consider the candidate Lyapunov function $V=$ $\frac{1}{2}\left(\tilde{x}_{1}^{2}+\tilde{x}_{2}^{2}+\tilde{x}_{3}^{2}\right)$. In $D^{+}$, the time derivative of $V$ is

$$
\dot{V}=\left(\begin{array}{c}
\tilde{x}_{1} \\
\tilde{x}_{2}
\end{array}\right)^{T} A\left(\begin{array}{c}
\tilde{x}_{1} \\
\tilde{x}_{2}
\end{array}\right)+\left(\begin{array}{c}
\tilde{x}_{1} \\
\tilde{x}_{2}
\end{array}\right)^{T} g\left(\tilde{x}_{1}, \tilde{x}_{2}, \tilde{x}_{3}\right) \tilde{x}_{3}-k \tilde{x}_{3}^{2}
$$

where $A=\left(\begin{array}{cc}-C_{g} a & C_{g} \frac{\bar{y}}{\bar{x}_{2}} \\ \frac{C_{g} a}{\bar{x}_{3}} & -\frac{w_{l, i n}}{\bar{x}_{3}}-C_{g} \frac{\bar{y}}{\bar{x}_{3} \bar{x}_{2}}\end{array}\right)$, and $g\left(\tilde{x}_{1}, \tilde{x}_{2}, \tilde{x}_{3}\right)=$ $\left(\begin{array}{c}{\left[C_{g} \frac{g \sin \theta}{A}+b \frac{\tilde{x}_{2}+\bar{x}_{2}}{\left(m_{3}^{\Delta}-\bar{x}_{3}-\tilde{x}_{3}\right)\left(m_{3}^{\Delta}-\bar{x}_{3}\right)}\right]^{\tilde{x}_{3}}\left[-\frac{C_{g} a}{\bar{x}_{3}} \tilde{x}_{1}+\frac{1}{\bar{x}_{3}}\left(w_{l, i n}+\frac{C_{g} \bar{y}}{\bar{x}_{2}}\right) \tilde{x}_{2}\right.} \\ \left.+C_{g} \frac{g \sin \theta}{A}+b \frac{\tilde{x}_{2}+\bar{x}_{2}}{\left(m_{3}^{\Delta}-\tilde{x}_{3}-\tilde{x}_{3}\right)\left(m_{3}^{\Delta}-\bar{x}_{3}\right)}\right]\end{array}\right)$. We now use the fact, proved in Appendix VII, that $g$ is Lipschitz on some compact set $K$, which yields

$$
\begin{aligned}
\left(\begin{array}{c}
\tilde{x}_{1} \\
\tilde{x}_{2}
\end{array}\right)^{T} g\left(\tilde{x}_{1}, \tilde{x}_{2}, \tilde{x}_{3}\right) & \leq \frac{1}{2}\left(\tilde{x}_{1}^{2}+\tilde{x}_{2}^{2}\right)+\frac{1}{2}\left\|g\left(\tilde{x}_{1}, \tilde{x}_{2}, \tilde{x}_{3}\right)\right\|^{2} \\
& \leq \frac{1}{2}\left(\tilde{x}_{1}^{2}+\tilde{x}_{2}^{2}\right)+\frac{L^{2}}{2}\left(\tilde{x}_{1}^{2}+\tilde{x}_{2}^{2}+\tilde{x}_{3}^{2}\right)
\end{aligned}
$$

Therefore, $\quad \dot{V} \leq-\left|\lambda_{\min }\right|\left(\tilde{x}_{1}^{2}+\tilde{x}_{2}^{2}\right)+\frac{\left|\tilde{x}_{3}\right|}{2}\left(L^{2}+1\right)\left(\tilde{x}_{1}^{2}+\tilde{x}_{2}^{2}\right)$

$$
-\left(k-L^{2} \frac{\left|\tilde{x}_{3}\right|}{2}\right) \tilde{x}_{3}^{2}
$$

where $\lambda_{\min }$ is the smallest (in absolute value) eigenvalue of $A$, which is negative as $A$ is obviously Hurwitz. Let now $c_{0} \in \mathbb{R}$ be $c_{0}=\sup \left\{c \in \mathbb{R} \mid\{V(x) \leq c\} \subset D^{+}\right\}$, and note $\Gamma=\left\{V(x) \leq c_{0}\right\}$ is the largest level set of $V$ contained in the region where the $\max$ functions are strictly positive. We also consider the set $\Omega=K \cap \Gamma \cap\left\{\left|\tilde{x}_{3}\right| \leq \frac{2\left|\lambda_{\min }\right|}{1+L^{2}}\right\}$. We claim that, provided that $k>\frac{\left|\lambda_{\min }\right| L^{2}}{1+L^{2}}, \Omega$ is positively invariant and contained in the basin of attraction of $(0,0,0)$. Indeed, inside $\Omega$, we have $\dot{\tilde{x}}_{3} \leq 0$ and (8) shows that we have $\dot{V} \leq 0$. Thus, any trajectory starting in $\Omega$ remains in $\Omega$ for all future times. As $\dot{V}$ is negative in $\Omega$, this shows, by LaSalle's theorem, that any trajectory starting in $\Omega$ converges to $\left(\tilde{x}_{1}, \tilde{x}_{2}, \tilde{x}_{3}\right)=(0,0,0)$. 


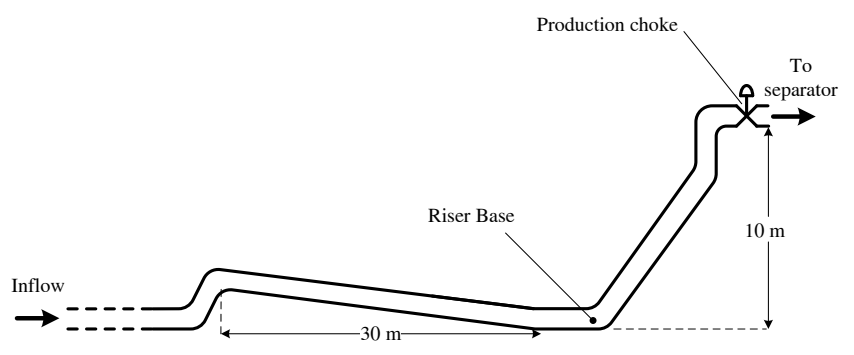

Fig. 2. Geometry of the experimental setup

Remark: The difficulty of this proof lies in the restriction to the set $D^{+}$. Indeed, if the dynamics in this set were valid at all times, one could easily prove the global asymptotic stability of the closed-loop system, by considering the third state $\tilde{x}_{3}$ as a decaying input for the subsystem $\left(\tilde{x}_{1}, \tilde{x}_{2}\right)$, and applying an Input-to-State Stability result. Yet, the presence of the $\max (\cdot, 0)$ functions in the equations makes this impossible, as it is impossible to guarantee that the trajectories remain in $D^{+}$. However, it is possible to prove that the trajectories which leave $D^{+}$eventually re-enter this set after some time. Physically, this means that the virtual valve cannot remain closed indefinitely. Also, the restriction of the basin of attraction to $\left\{\left|\tilde{x}_{3}\right| \leq \frac{2\left|\lambda_{\min }\right|}{1+L^{2}}\right\}$ could be avoided, as the trajectories always enter this set after a certain time (depending on the initial condition), given the exponential convergence of $\tilde{x}_{3}$.

\section{EXPERIMENTS}

In order to evaluate the performances of the controller designed in the previous section, we conducted experiments on a multiphase flow loop in the Statoil Research Center located in Porsgrunn, Norway. The main results of these experiments are presented here.

\section{A. Experimental setup}

Installations: The multiphase flow loop consists in a 115 meter-long, 3 inch-thick pipe, filled with air and water (replacing the gas and oil), and reproduces the qualitative behavior of offshore oil fields. In particular, under certain conditions on the inflow rates of air and water, this experimental test rig can reproduce the slugging phenomenon. The geometry of the system is as depicted in Figure 2. After being injected in the pipe from the reservoirs thanks to pumps, the fluids have to go through a 30 meter-long declining ramp, followed by a short horizontal section that precedes the riser, where the average of the slope is approximatively $45^{\circ}$. At the top of the riser, the fluids pass through the automated production choke, and enter a separator where the pressure is set to a constant value.

On the experimental setup, pressure sensors are located along the pipe. Only two of them were used for the experiments: the Riser Base Pressure (RBP) sensor was used for the PI controller, whereas a measurement of the pressure at the top of the riser, upstream the production choke was used for the model-based controller.
Setup: The inflow rates were chosen to be $26 \mathrm{~kg} / \mathrm{h}$ for the air and $4 \mathrm{~m}^{3} / \mathrm{h}$ for the water. The separator pressure was chosen to be the atmospheric pressure. The inflows were stabilized (as tightly as possible) to a constant value thanks to PI controllers acting on the injection pumps. With this setup, the period of the slugging is close to $100 \mathrm{~s}$, with a RBP oscillating (roughly) between 1.4 bar and 2.2 bar (the magnitude and frequency depend on the operating point considered). The practical bifurcation point corresponded to a $25 \%$ choke opening, which means that, for lower choke openings, the slugging phenomenon disappears. Nevertheless, even for these stable operating points, residual pressure oscillations are observed. They correspond to hydrodynamic effects, and have much higher frequency $(\approx 5 \mathrm{~s})$ and lower amplitude than the oscillations due to slugging.

\section{B. Results}

In this section, we compare the performances of the PI controller and the model-based controller. On an offshore field, the performances would be assessed in terms of oil recovery: the best controller would be the one yielding the less production losses. However, in the case of the experimental flow loop considered here -as well as many other experimental facilities-, the inflows are kept constant (to isolate multi-phase flow effects from other effects). Therefore, inflow rates do not depend on the operating point. At steady-state, the production flow rates of oil and gas are equal to the inflow rates, which means that it is impossible to see the effect of the controllers in terms of oil recovery. The right variable to consider to evaluate the performances is then the value of the equilibrium Riser Base Pressure. The lower this value is, the harder it is to stabilize the flow. Also, a lower steady-state RBP will yield a higher production on an offshore field.

1) PI controller: We first tried to stabilize the flow around an unstable point using a PI feedback loop on the RBP. As mentionned in Section II, this required a great tuning effort, and only allowed us to suppress the slugging around an operating point very close to the bifurcation. Figure 3 plots the values of the RBP and the choke opening over time. The pressure setpoint was $R B P_{\text {ref }}=1.9$ bar, which corresponds to a $26 \%$ choke opening. For comparison, the equilibrium RBP corresponding to a $25 \%$ choke opening (that is to say at the bifurcation point) is 1.95bar. For lower values of the Riser Base Pressure setpoint, the PI controller failed to stabilize the flow, despite a tedious tuning effort. Also, for the same setpoint, the controller failed to stabilize the system when started at a different phase of the slugging cycle. This confirms the weaknesses of the PI controller mentionned in Section II.

2) Model-based controller: Using the model-based controller proposed in Theorem 4.1 allowed us to reach unstable operating points significantly above (in terms of choke opening) the stability limit. Figure 4 shows the values of the $\mathrm{RBP}$ and the choke opening for this experiment. The pressure setpoint was $R B P_{\text {ref }}=1.78$ bar, which corresponds to a $36 \%$ equilibrium choke opening. 


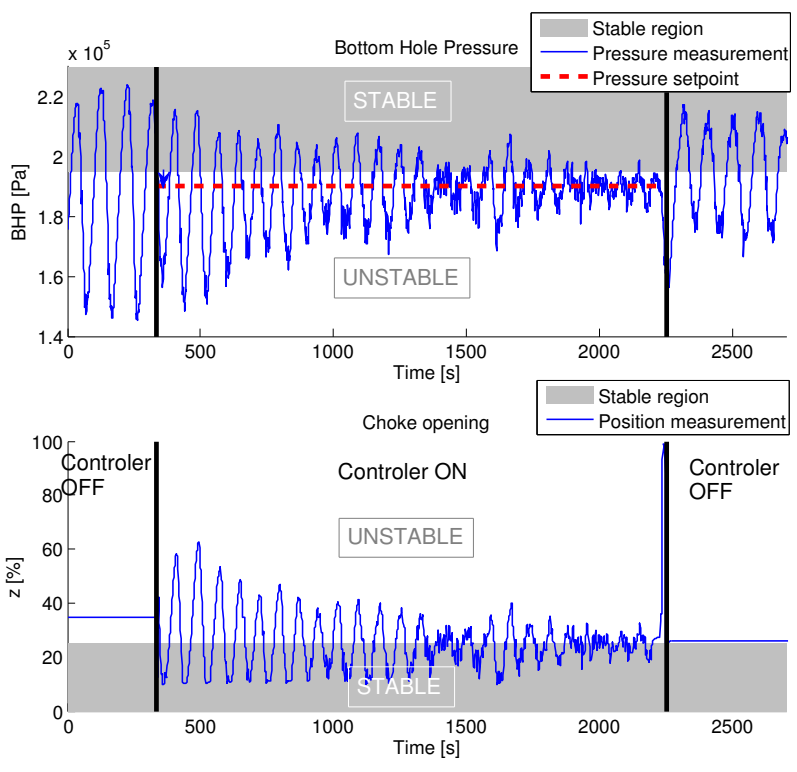

Fig. 3. Stabilization to $R B P_{\text {ref }}=1.9$ bar with PI controller

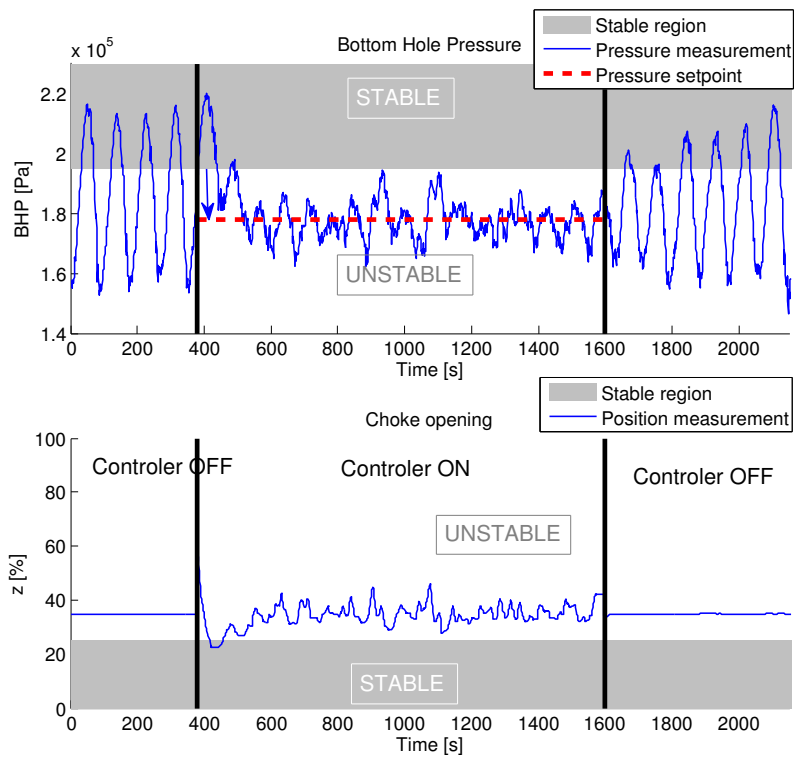

Fig. 4. Stabilization with model-based controller. As illustrated by the arrow, the equilibrium RBP is far below the stability limit, contrary to the results obtained with the PI (in Figure 3). This equilibrium is more difficult to reach, and would yield a higher production on a real field.

a) Remark: Equation (7) indicates that the control law requires a mass of liquid setpoint $\bar{x}_{3}$. The mass setpoint corresponding to the desired pressure setpoint can be found through the model, or adapted with a slow integrator loop. In the case of Figure 4, no integrator was used. The evolution of the estimated mass of liquid in the riser $m_{l, r}$, converging exponentially to its setpoint $\bar{x}_{3}=8.7 \mathrm{~kg}$ is plotted in Figure 5 . The model-based controller was able to stabilize the flow for higher equilibrium values of the RBP, that is to say, closer to the stability limit. Stability was achieved in all cases regardless of what the state of the system was when we turned the controller on.

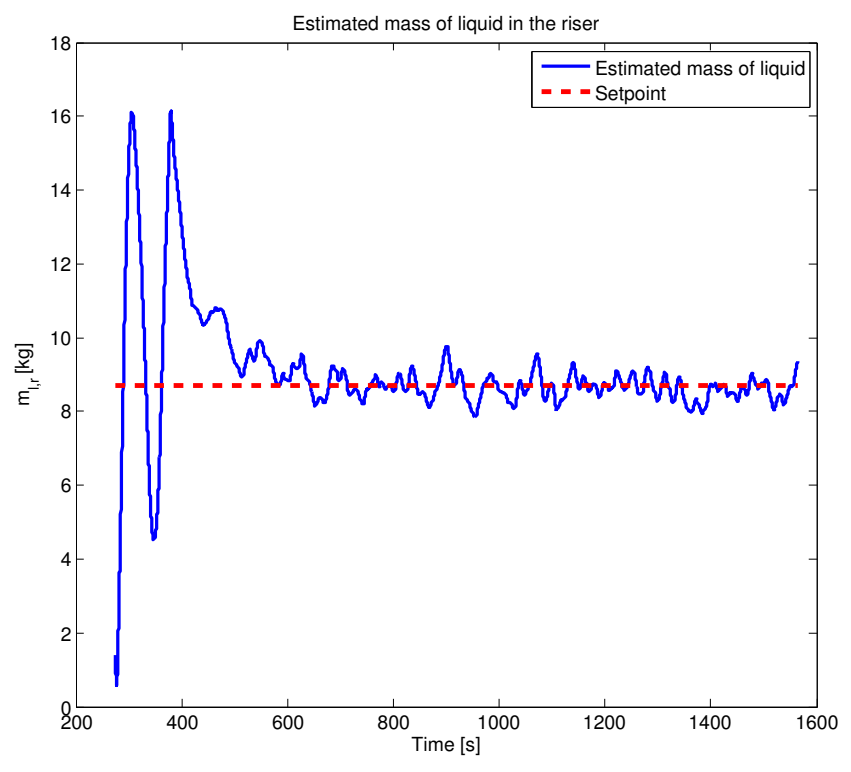

Fig. 5. Convergence of the estimated mass of liquid in the riser.

\section{CONCLUSIONS AND FUTURE WORK}

In the continuity of [14], we have presented an innovative solution to cope with slug flow. The model was used to derive a control law which proves more efficient than the conventional PI controllers. A theoretical proof of convergence of the control scheme is given, as well as a mid-scale experimental validation of this result. The experiments show that the model-based controller is less sensitive to changes in operating conditions, and allows stabilization for larger choke openings than the PI controller. Further, the good performances of the model-based controller suggest that, rather than the RBP, the right variable to control may be the mass of liquid in the riser. These two variables are closely related, as the mass of liquid is, in the model, proportional to the pressure drop over the riser $\triangle P_{\text {riser }}=R B P-U C P$ (where $U C P$ is the Upstream Choke Pressure, denoted $y$ in the model). This results from the simplification we made by assuming that the pressure drop over the riser was simply due to gravity, thus neglecting friction. To verify whether the mass of liquid is a better variable to consider for feedback control than the RBP, we aim to perform more experiments, using a simple PI controller on $\Delta P_{\text {riser }}$. Yet, this requires a proper filter, as the UCP measurement is usually very noisy. Candidate filters include a first-order low-pass filter, or simply the observed mass of liquid given by our model (with the right scaling factor).

Another feature of this controller, which was not discussed here due to a lack of space, is that it does not require any RBP measurement. Indeed, as mentioned in Section IV, the controller uses an observer to perform full-state feedback. The observer we designed only uses the UCP measurement, which is more frequently available on offshore fields, and more reliable than the RBP sensors. Details on this observer, as well as a full proof of convergence of the closed-loop system with the observer will be the subject of a future paper. 


\section{REFERENCES}

[1] A. M. Ansari, U. Sylvester, O. Shoham, and U. Brill, "A comprehensive mechanistic model for upward two-phase flow in wellbores." $S P E$ Annual Technical Conference, 1990.

[2] A. B $\emptyset$ e, "Severe slugging characteristics ; part i:flow regime for severe slugging ; part ii: Point model simulation study." Presented at Selected Topics in Two-Phase Flow, NTH, Trondheim, Norway, 1986.

[3] A. E. Dukler and M. G. Hubbard, "A model for gas-liquid slug flow in horizontal and near horizontal tubes," Industrial $\mathcal{E}$ Engineering Chemistry Fundamentals, vol. 14, no. 4, pp. 337-347, 1975.

[4] E. Duret, "Dynamique et contrôle des écoulements polyphasiques," Ph.D. dissertation, Ecole des Mines de Paris, 2005.

[5] R. C. Fernandes, R. Semiat, and A. E. Dukler, "Hydrodynamic model for gas-liquid slug flow in vertical tubes," AIChE Journal, vol. 29, pp. 981-989, 1983.

[6] E. Storkaas, "Control solutions to avoid slug flow in pipeline-riser systems," Ph.D. dissertation, Norwegian University of Science and Technology, 2005.

[7] Y. Taitel and D. Barnea, "Two-phase slug flow," Advances in Heat Transfer, vol. 20, pp. 83 - 132, 1990

[8] B. Hu, "Characterizing gas-lift instablilities," Ph.D. dissertation, Department of Petroleum Engineering and Applied Geophysics, NTNU, 2004.

[9] L. Sinègre, "Dynamic study of unstable phenomena stepping in gaslift activated systems," Ph.D. dissertation, Ecole des Mines de Paris, 2006

[10] J.-M. Godhavn, M. P. Fard, and P. H. Fuchs, "New slug control strategies, tuning rules and experimental results," Journal of Process Control, vol. 15, pp. 547-557, 2005.

[11] A. Courbot, "Prevention of severe slugging in the dunbar 16' multiphase pipeline," Offshore Technology Conference, 1996.

[12] K. Havre and M. Dalsmo, "Active feedback control as the solution to severe slugging," SPE Annual Technical Conference, 2001.

[13] V. Henriot, E. Duret, E. Heintz, and A. Courbot, "Multiphase production control: Application to slug flow," Oil E Gas Science and Technology - Rev IFP, vol. 57, No. 1, pp. 87-98, 2002.

[14] F. Di Meglio, G.-O. Kaasa, and N. Petit, "A first principle model for multiphase slugging flow in vertical risers," Conference on Decision and Control, 2009.

[15] F. E. Jansen, O. Shoham, and Y. Taitel, "The elimination of severe slugging-experiments and modeling," International Journal of Multiphase Flow, vol. 22, no. 6, pp. 1055 - 1072, 1996.

[16] Z. Schmidt, J. Brill, and H. Beggs, "Choking can eliminate severe pipeline slugging," Oil $\mathcal{E}$ Gas Journal, vol. 12, pp. 230-238, 1979.

[17] V. Henriot, A. Courbot, E. Heintze, and L. Moyeux, "Simulation of process to control severe slugging: Application to the dunbar pipeline," SPE Annual Technical Conference, 1999

[18] B. Jansen, M. Daslmo, L. Nøkleberg, K. Havre, V. Kristiansen, and P. Lemetayer, "Automatic control of unstable gas lifted wells," SPE annual technical conference, 1999.

[19] M. Dalsmo, E. Halvorsen, and O. Slupphaug, "Active feedback control of unstable wells at the brage field," SPE Annual Technical Conference, 2002 .

[20] B. Hu and M. Golan, "Gas-lift instablility resulted production loss and its remedy by feedback control: Dynamical simulation results," $S P E$, 2003

[21] G. O. Eikrem, L. Imsland, and B. Foss, "Stabilization of gas-lifted wells based on state estimation," International Symposium on Advanced Control of Chemical Processes, 2004.

\section{Proof of Lipschitzness}

Let us first prove that the compact set $K=\left[x_{1}^{-}, x_{1}^{+}\right] \times$ $\left[x_{2}^{-}, x_{2}^{+}\right] \times\left[x_{3}^{-}, x_{3}^{+}\right]$is positively invariant for (4)-(5)-(6), with

$$
\begin{array}{rlrl}
x_{1}^{-}=\frac{m_{3, \text { still }}}{a} \frac{g \sin \theta}{A} & x_{1}^{+} & =\frac{1}{C_{g} a}\left[-\epsilon w_{g, \text { in }}+\frac{\bar{y}}{b} \frac{1-\mu}{\mu} w_{l, \text { in }}\right] \\
& +\frac{\bar{y}}{a}+\left(\mu m_{3}^{\Delta}+m_{3, \text { still }}\right) \frac{g \sin \theta}{a A} \\
x_{2}^{-}=\frac{\bar{y}}{b} \frac{\eta}{1-\eta} & x_{2}^{+}=\frac{\bar{y}}{b} \frac{1-\mu}{\mu} \\
x_{3}^{-}=\mu m_{3}^{\Delta} & x_{3}^{+}=(1-\eta) m_{3}^{\Delta}
\end{array}
$$

and $0<\eta<1$ and $0<\mu<1$ are two parameters chosen such that

$$
\eta<\frac{b \epsilon G L R}{\bar{y}+b \epsilon G L R}, \quad \eta+\mu<1
$$

and

$$
\begin{array}{r}
\eta \mu w_{g, i n}-\frac{w_{l, i n}}{b} \bar{y}(1-\mu) \eta-C_{g} \bar{y}(1-\mu-\eta) \\
+C_{g} \frac{g \sin \theta}{A} m_{3}^{\Delta}(1-\mu-\eta) \eta \mu \leq 0
\end{array}
$$

where $G L R=\frac{w_{g, i n}}{w_{l, i n}} .{ }^{1}$ We first prove that for $x_{1}=x_{1}^{-}$, we have $\dot{x}_{1}>0$. Indeed, for any $\left(x_{2}, x_{3}\right) \in\left[x_{2}^{-}, x_{2}^{+}\right] \times\left[x_{3}^{-}, x_{3}^{+}\right]$

$$
\begin{aligned}
\dot{x}_{1}\left(x_{1}^{-}, x_{2}, x_{3}\right) & =(1-\epsilon) w_{g, \text { in }} \\
& -C_{g} \max \left(-b \frac{x_{2} x_{3}}{m_{3}^{\Delta}-x_{3}}-x_{3} \frac{g \sin \theta}{A}, 0\right) \\
& =(1-\epsilon) w_{g, \text { in }}>0
\end{aligned}
$$

Let us now prove that $\dot{x}_{3}\left(x_{3}^{+}\right) \leq 0$. For any $x_{2} \in\left[x_{2}^{-}, x_{2}^{+}\right]$, we have

$$
\begin{aligned}
\dot{x}_{3}\left(x_{3}^{+}\right) & =w_{l, i n}-u C_{c} \rho_{l}\left(b x_{2} \frac{1-\mu}{\mu}-p_{s}\right)^{1 / n} \\
& \leq w_{l, i n}-u C_{c} \rho_{l}\left(b x_{2}^{-} \frac{1-\mu}{\mu}-p_{s}\right)^{1 / n} \leq 0
\end{aligned}
$$

Similarly, it is easy to prove that $\dot{x}_{3}\left(x_{3}^{-}\right) \geq 0$ using the definition of $x_{2}^{+}$. It is also easy to show that $\dot{x}_{2}\left(x_{2}^{-}\right) \geq 0$, as follows

$$
\dot{x}_{2}\left(x_{1}, x_{2}^{-}, x_{3}\right) \geq \frac{1}{x_{3}}\left[\epsilon w_{g, i n}-x_{2}^{-} w_{l, i n}\right]>0
$$

thanks to (9) and the definition of $x_{2}^{-}$. Similarly, using the definition of $x_{1}^{+}, x_{2}^{+}$and $x_{3}^{-}$, it is easy to show that $\dot{x}_{2}\left(x_{2}^{+}\right) \leq 0$. Eventually, we prove that $\dot{x}_{1}\left(x_{1}^{+}\right) \leq 0$ by using (10)

$$
\begin{aligned}
\dot{x}_{1}\left(x_{1}^{+}, x_{2}, x_{3}\right) & \leq(1-\epsilon) w_{g, \text { in }}-C_{g} \max \left[a x_{1}^{+}-b \frac{x_{2}^{+} x_{3}^{+}}{m_{3}^{\Delta}-x_{3}^{+}}\right. \\
& \left.-\left(x_{3}^{+}+m_{3, \text { still }}\right) \frac{g \sin \theta}{A}, 0\right] \\
& \leq w_{g, \text { in }}-\frac{\bar{y}}{\mu}\left[\frac{w_{l, \text { in }}}{b}(1-\mu)-C_{g} \frac{1-\mu-\eta}{\eta}\right] \\
& +C_{g}(1-\mu-\eta) \frac{g \sin \theta}{A} m_{3}^{\Delta}
\end{aligned}
$$

which is negative according to (10). These inequalities show that $K$ is positively invariant. Besides, inside the compact set $K$, the function $g$ defined in the proof of Theorem 4.1 is $C^{1}$, therefore it is globally $L$-Lipschitz on $K$ for some constant $L$.

\footnotetext{
${ }^{1}$ Inequations (9) and (10) are verified for $\eta=\mu=0$. Even though the two parameters must be strictly positive, this guarantees, by continuity, their existence. In particular, they can be taken as close to 0 as one wants.
} 
VIII. Equilibrium of SYSTEM (4)-(5)-(6)

The equilibrium of system (4)-(5)-(6) is given by

$$
\begin{aligned}
& \bar{x}_{1}(\bar{u})=(1-\epsilon) \frac{w_{g, i n}}{C_{g}}+\frac{\bar{y}(\bar{u})}{a}+\left(\bar{x}_{3}(\bar{u})+m_{3, s t i l l}\right) \frac{g \sin \theta}{A} \\
& \bar{x}_{2}(\bar{u})=\frac{w_{g, i n}}{w_{l, i n}} \\
& \bar{x}_{3}(\bar{u})=\frac{\bar{y}(\bar{u})}{\bar{y}(\bar{u})+b G L R} m_{3}^{\Delta}, \text { with } \bar{y}(\bar{u})=p_{s}+\left(\frac{w_{l, i n}}{\bar{u} C_{c} \rho_{l}}\right)^{n} .
\end{aligned}
$$

